\title{
Controlled Nanoparticle Targeting and Nanoparticle-Driven Nematic Structural Transition
}

\author{
A. V. Dubtsov, ${ }^{1}$ S. V. Pasechnik, ${ }^{1}$ D. V. Shmeliova, ${ }^{1}$ S. Kralj, ${ }^{2}$ and R. Repnik ${ }^{2}$ \\ ${ }^{1}$ Moscow State University of Instrument Engineering and Computer Science, Stromynka 20, Moscow 107996, Russia \\ ${ }^{2}$ Faculty of Natural Sciences and Mathematics, University of Maribor, Koroska 160, SI-2000 Maribor, Slovenia
}

Correspondence should be addressed to R. Repnik; robert.repnik@um.si

Received 21 November 2014; Revised 25 December 2014; Accepted 19 January 2015

Academic Editor: Ivan Smalyukh

Copyright (C) 2015 A. V. Dubtsov et al. This is an open access article distributed under the Creative Commons Attribution License, which permits unrestricted use, distribution, and reproduction in any medium, provided the original work is properly cited.

We study experimentally and theoretically controlled targeting of specific nanoparticles (NPs) to different regions within nematic liquid crystal. Using a simple mesoscopic Landau-de Gennes-type model in terms of a tensor nematic order parameter, we demonstrate a general mechanism which could be exploited for controlled targeting of NPs within a spatially nonhomogeneous nematic texture. Furthermore, we experimentally demonstrate using polarising microscopy that even a relatively low concentration of localised appropriate NPs could trigger a nematic structural transition. A simple estimate is derived to account for the observed transition.

\section{Introduction}

Recent years witness increased interest in soft nanocomposites consisting of soft matrices hosting specific nanoparticles (NPs) [1-3]. Appropriate combinations could yield quantitatively dramatically enhanced or qualitatively new behaviour that individual components do not exhibit on their own. Such systems are promising to trigger development of new nanobased practical applications. Furthermore, they could be also exploited as convenient testing ground for basic physics [1].

As soft matrices various liquid crystal (LC) phases and structures are often used [4-7]. In addition to softness (i.e., capability to exhibit strong response to even weak local perturbation), they are also optically transparent and in many cases stable close to room temperatures. Among others, these properties make them relatively easily accessible for experimental observations [5-7]. When various NPs are introduced to soft matrices several scenarios could be realized. In most cases it is of interest to obtain homogeneous mixtures of LCs and NPs and to avoid phase segregation or agglomeration of NPs [3]. If spatial nonhomogeneities exist within LC matrix, in most cases NPs tend to assemble in regions exhibiting relatively strong elastic distortions [1]. Furthermore, LC mediated interactions among NPs could enable their selforganization into different tunable superstructures [3].
In this paper, we study numerical targeting of specific NPs towards desired locations within spatially inhomogeneous nematic LC structures. Furthermore, we demonstrate experimentally that a relatively low concentration of localised NPs could be sufficient to trigger a global nematic structural transition.

The plan of the paper is as follows. In Section 2 we present the Landau-de Gennes mesoscopic model. In Section 3 we describe experimental setup. In Section 4 we theoretically study interactions between NPs and local LC ordering focusing on NP surface treatment. In Section 5 we present our experimental results demonstrating a nanoparticle-driven structural transition. A simple estimate is presented to explain the observed transition. In the last section, we summarize our findings. Some technical details are given in the appendix.

\section{Mesoscopic Modelling}

At mesoscopic level, we describe a local nematic order in terms of the traceless and symmetric tensor order parameter $[7,8]$ as

$$
\mathbf{Q}=\sum_{i=1}^{3} s_{i} \vec{e}_{i} \otimes \vec{e}_{i} .
$$


The quantities $s_{i}$ and unit vectors $\vec{e}_{i}$ determine eigenvalues and eigenvectors of $\mathbf{Q}$, respectively. In case of uniaxial order, $\mathbf{Q}$ is commonly expressed as [7, 8]

$$
\mathbf{Q}=S\left(\vec{n} \otimes \vec{n}-\frac{\mathbf{I}}{3}\right) .
$$

Here, I is the identity tensor, $\vec{n}$ describes the nematic director field, and $S$ is the scalar uniaxial order parameter field. The unit vector field $\vec{n}$ points along the local uniaxial ordering, where states $\pm \vec{n}$ are physically equivalent. Furthermore, $S$ quantifies the extent of fluctuations about $\vec{n}$. A local degree of biaxiality in $\mathbf{Q}$ is measured by the biaxial parameter [9]

$$
\beta^{2}=1-\frac{6\left(\operatorname{tr} \mathbf{Q}^{3}\right)^{2}}{\left(\operatorname{tr} \mathbf{Q}^{2}\right)^{3}}
$$

and $\beta^{2} \in[0,1]$. Uniaxial ordering corresponds to $\beta^{2}=0$ and maximal degree of biaxiality to $\beta^{2}=1$.

The resulting free energy $[7,8]$ is expressed as

$$
F=\iiint\left(f_{c}+f_{e}\right) d^{3} \vec{r}+\iint f_{i}^{(\mathrm{con})} d^{2} \vec{r}+\iint f_{i}^{(\mathrm{NP})} d^{2} \vec{r},
$$

where $f_{c}, f_{e}, f_{i}^{(\mathrm{con})}$, and $f_{i}^{(\mathrm{NP})}$ stand for the condensation, elastic, confinement, and LC-NP interface free density contributions, respectively. The first integral is carried out over the $\mathrm{LC}$ volume. The second integral is performed over the surface area enclosing LC body and the third one over the NP-LC interface. In the lowest order approximation, which is needed to explain phenomena of our interest, they are expressed as

$$
\begin{gathered}
f_{c}=\frac{A_{0}\left(T-T_{*}\right)}{2} \operatorname{tr} \mathbf{Q}^{2}-\frac{B}{3} \operatorname{tr} \mathbf{Q}^{3}+\frac{C}{4}\left(\operatorname{tr} \mathbf{Q}^{2}\right)^{2}, \\
f_{e}=\frac{L}{2} \operatorname{tr}(\nabla \mathbf{Q})^{2} \\
f_{i}^{(\text {loc })}=w^{(\text {loc })} \vec{v} \cdot \mathbf{Q} \cdot \vec{v} .
\end{gathered}
$$

The condensation term enforces uniaxial nematic ordering. The quantities $A_{0}, B$, and $C$ are material constants and $T_{*}$ is the bulk isotropic phase supercooling temperature. The elastic term is weighted by the positive elastic constants $L$ which tends to enforce homogenous ordering in $\mathbf{Q}$. The interface term, where the superscript loc stands for location, determines conditions at the LC confining interface (loc = con) or at the NP-LC interface (loc $=$ NP). The quantity $w^{(\text {loc })}$ is a surface strength constant, and $\vec{v}$ is a surface normal unit vector [4]. For an interface enforcing homeotropic (isotropic tangential) ordering it holds $w^{(\text {loc })}<0\left(w^{(\text {loc })}>0\right)$.

\section{Experimental Setup}

In the experimental part of work, we used the nematic mixture LC ZhK 616. It consists of p-n-butyl-p-methoxyazoxybenzene (59\%), p-n-butyl-p-heptanoylazoxybenzene (29\%), and n-heptyl-benzoic acid n-cyano-phenyl ester (12\%). The mixture possesses an optical anisotropy $\Delta n=0.23$ and relatively wide nematic phase window extending within the temperature interval $\sim\left[0,70^{\circ} \mathrm{C}\right]$.

Microdroplets of LC were dispersed in deionized water with a resistivity of $18.2 \mathrm{M} \Omega \mathrm{cm}$. The $\mathrm{LC}$ emulsion in water was formed by sequential cycles of sonication and vortex mixing of $2 \mu \mathrm{L}$ of $\mathrm{LC}$ and $2 \mathrm{~mL}$ of water at room temperature. Powdered phospholipid 1,2-diacyl-sn-glycero-3phosphocholine (L- $\alpha$-phosphatidylcholine, SIGMA Aldrich purity $>99 \%$ ) was dispersed in deionized water at room temperature. Dispersion of the phospholipid powder in water at initial concentration of $10^{-3} \mathrm{~g} / \mathrm{mL}$ was mixed by vortexing. Different dilutions were made to obtain concentrations ranging from $10^{-4} \mathrm{~g} / \mathrm{mL}$ to $10^{-8} \mathrm{~g} / \mathrm{mL}$. A volume of $4 \mu \mathrm{L}$ of an aqueous dispersion of phospholipid was dispensed onto a glass substrate. A volume of $4 \mu \mathrm{L}$ of an LC emulsion was added to the water dispersion of the phospholipid. A polarized microscope with an objective of 100x magnification was used to monitor nematic order within LC droplets of diameters between $1 \mu \mathrm{m}$ and $10 \mu \mathrm{m}$.

\section{Interaction between a Surface Treated Nanoparticle and Local Nematic Order}

In this section we demonstrate a robust and simple NP targeting mechanism which could be exploited to assemble NPs within a desired region. For this purpose we study theoretically the impact of NP's surface coating on interaction with its nematic surrounding using a simple confinement displaying cylindrical symmetry which can stabilize NLC structures displaying three distinctively different characteristic local nematic textures.

The geometry of the problem is sketched in Figure 1(a). We consider a nanoparticle immersed within a plan-parallel hybrid cell of thickness $h$ and radius $R$. For the sake of simplicity, which does not affect general validity of results obtained, both the nanoparticle and the cell geometry exhibit the cylindrical symmetry. The cylindrical coordinate system is defined with the orthonormal triad of unit vectors $\left\{\vec{e}_{\rho}, \vec{e}_{\varphi}, \vec{e}_{z}\right\}$.

At the top plate $(z=h)$ and at the bottom plate $(z=$ 0 ) we impose strong uniaxial radial anchoring and strong homeotropic anchoring, respectively. Therefore, we enforce

$$
\begin{aligned}
& \mathbf{Q}(\rho, \varphi, z=h)=S_{\mathrm{eq}}\left(\vec{e}_{\rho} \otimes \vec{e}_{\rho}-\frac{\mathbf{I}}{3}\right) \equiv \mathbf{Q}_{\mathbf{r}}, \\
& \mathbf{Q}(\rho, \varphi, z=0)=S_{\mathrm{eq}}\left(\vec{e}_{z} \otimes \vec{e}_{z}-\frac{\mathbf{I}}{3}\right) \equiv \mathbf{Q}_{\mathbf{h}} .
\end{aligned}
$$

Here, $S_{\text {eq }}$ stands for the bulk equilibrium value of $S$ minimizing the condensation free energy density. At the lateral sites, the free boundary condition is enforced. In such geometry the boojum topological defect is enforced at the top plate, the structure of which is studied in detail in [10]. For chosen boundary conditions it exhibits cylindrically symmetric finger-like defect core structure. The latter is well characterized with $\beta^{2}(\rho, z)$ spatial variation shown in Figure 1(b). We emphasized that the finger-tip, which is displaced from the top plate for a distance comparable with 


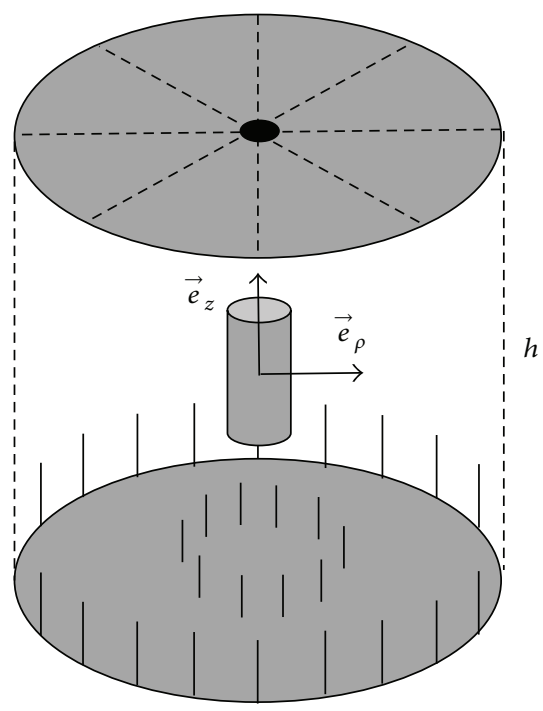

(a)

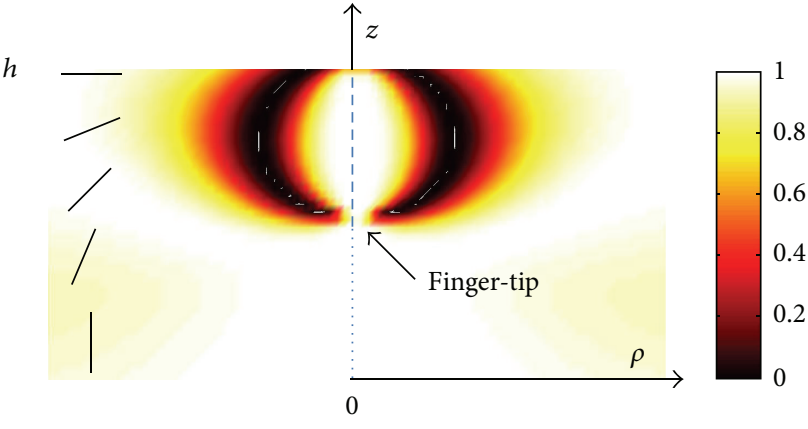

(b)

Figure 1: (a) Schematic presentation of the hybrid plan-parallel cell of thickness $h$ hosting a cylindrical NP. The diameter and the height of the NP are set to be equal to $\xi_{b} / 3 \sim 10 \mathrm{~nm}$ and $h=10 \xi_{b}$. (b) Cross section through the boojum is shown where we plot the degree of biaxiality $\beta^{2}$. On the left side of the figure we schematically sketch typical nematic director field variation along the $z$-axis away from the axis of symmetry. A biaxial shell joins the isotropic finger-tip and the upper surface. Along the cylindrical axis the system exhibits uniaxial ordering due to topological reasons. With dashed (dotted) line we mark regions exhibiting negative (positive) uniaxiality. In the illustration the anchoring strength at the top plane is finite. The characteristic defect size is comparable to the biaxial correlation length. The colour bar of $1-\beta^{2}$ is shown on the right side of the figure.

the biaxial correlation length $\xi_{b}$ (see the appendix), is due to topological reasons melted [10]. In typical LCs, $\xi_{b}$ roughly ranges between $20 \mathrm{~nm}$ and $40 \mathrm{~nm}[11]$.

Therefore, the chosen geometry enforces three distinctively different LC structures: (i) cylindrical radial-like profile close to the top boundary at $z=h$, (ii) essentially homogenous ordering along $\vec{e}_{z}$ at the bottom plate at $z=0$, and (iii) essentially melted region at the boojum finger-tip.

The mass point of a cylindrical NP of radius $r$ and height $2 r$ is placed at an arbitrary position $z=z_{\mathrm{NP}}$ at the axis of system's cylindrical symmetry. We consider different NP surface coatings. At NP-LC interfaces we enforce three qualitatively different surface conditions, to which we henceforth refer to as (i) radial, (ii) homogeneous, and (iii) melted interfaces. For these cases we impose at the interfaces (i) $\mathbf{Q}\left(\rho, \varphi, z_{\mathrm{NP}}-r \leq z \leq z_{\mathrm{NP}}+r\right)=\mathbf{Q}_{\mathbf{r}}$, (ii) $\mathbf{Q}\left(\rho, \varphi, z_{\mathrm{NP}}-\right.$ $\left.r \leq z \leq z_{\mathrm{NP}}+r\right)=\mathbf{Q}_{\mathbf{h}}$, and (iii) $\mathbf{Q}\left(\rho, \varphi, z_{\mathrm{NP}}-r \leq\right.$ $\left.z \leq z_{\mathrm{NP}}+r\right)=0$, respectively. For a fixed position of NP we calculate numerically the equilibrium NLC structure. Some technical details are presented in the appendix. Typical two-dimensional profiles $\beta^{2}(\rho, z)$ for different positions of NPs and the melted NP-LC interface condition are shown in Figure 2.

To calculate a mechanical force $\vec{F}_{m}$ on an object immersed in orientationally ordered LC medium we calculate the free energy of equilibrium LC structure for a NP fixed at $\vec{r}$. Then, we slightly displace the NP and recalculate the energy for new LC equilibrium structure. From the gradient of free energy we obtain the mechanical force experienced by the NP.
In Figure 3 we plot two-dimensional plots showing calculation details within the $\{\rho, z\}$ plane for a given position of a NP enforcing melted boundary condition. In Figure 3(a), we plot $\beta^{2}(\rho, z)$. The rim corresponding to $\beta^{2}(\rho, z)=1$ reveals states exhibiting maximal degree of biaxiality. Essentially uniaxial states, which are well described by the uniaxial tensor order parameter (see (2)), correspond to $\beta^{2}(\rho, z) \sim 0$. In Figure 3(b), we plot the angle $\theta(\rho, z)$ describing orientation of the principal eigenvector $\vec{e}_{1}=\vec{e}_{\rho} \sin \theta+\vec{e}_{z} \cos \theta$, corresponding to the largest $\mathbf{Q}$ eigenvalue within the $\{\rho, z\}$ plane. In case of uniaxial ordering it holds $\vec{e}_{1}=\vec{n}$. Note that we enforce $\theta=45^{\circ}$ in the region occupied by the NP in order to make it more visible. In Figure 3(c), we plot the degree of order $q(\rho, z)=\sqrt{\operatorname{Tr} \mathbf{Q}^{2}}$.

In Figure 4 we plot the force as a function of $z$-coordinate for the three different interfaces. One sees that a nanoparticle, which locally enforces (i) radial, (ii) melted, or (iii) homogeneous LC configuration, tends to assemble at (i) the top plate, (ii) the finger-tip, and (iii) the bottom plate, respectively. Therefore, NP tends to assemble in a region which matches best with its interface-enforced LC pattern.

\section{Nanoparticle-Driven Nematic Structural Transition}

In this section we demonstrate that relatively low concentration of appropriate NPs could trigger a nematic structural transition. For this purpose we study micrometer-sized NLC 


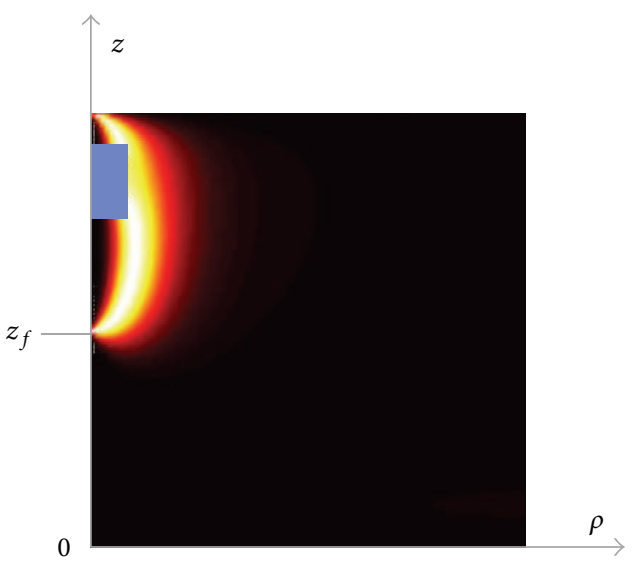

(a)

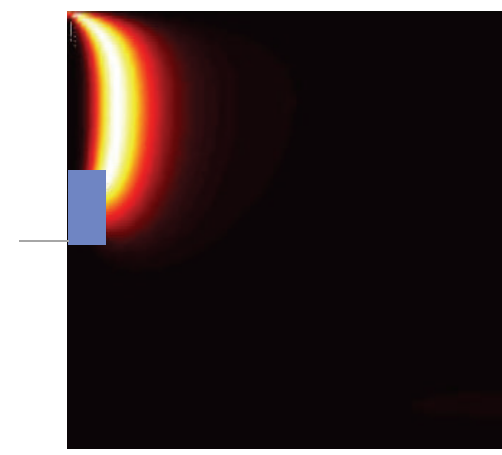

(d)

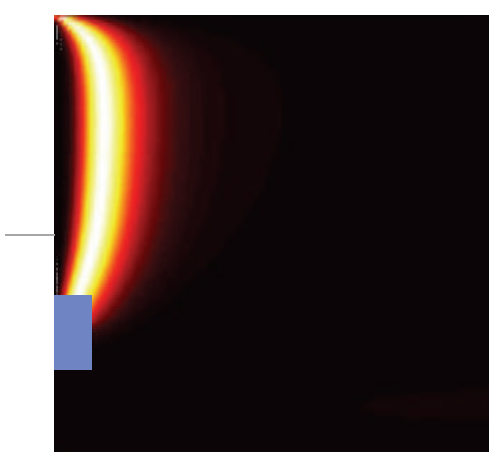

(g)

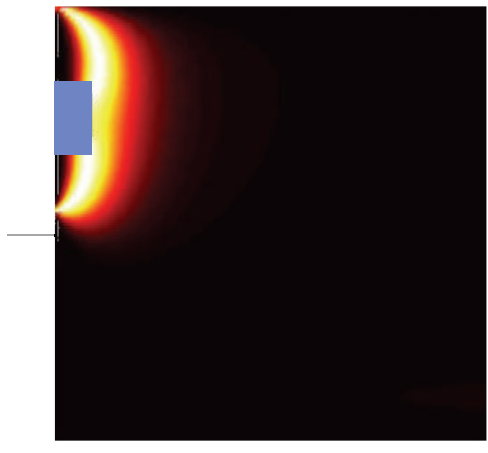

(b)

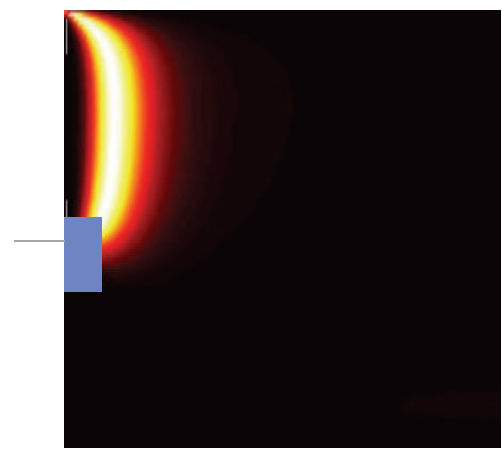

(e)

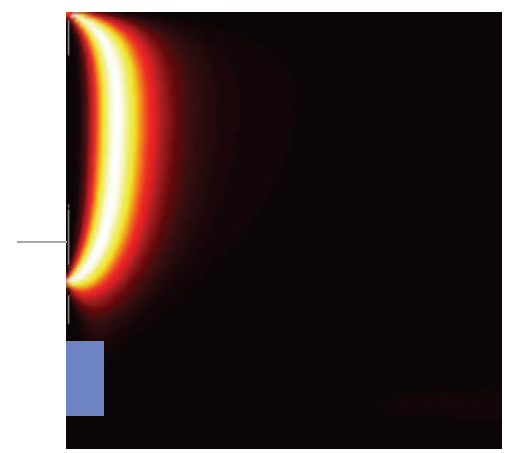

(h)

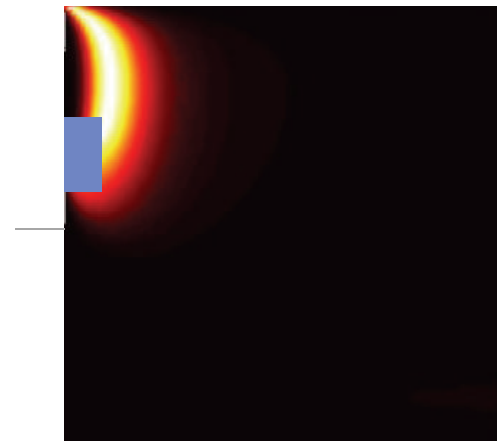

(c)

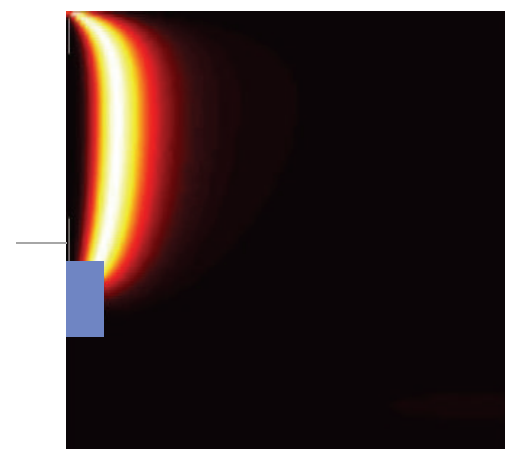

(f)

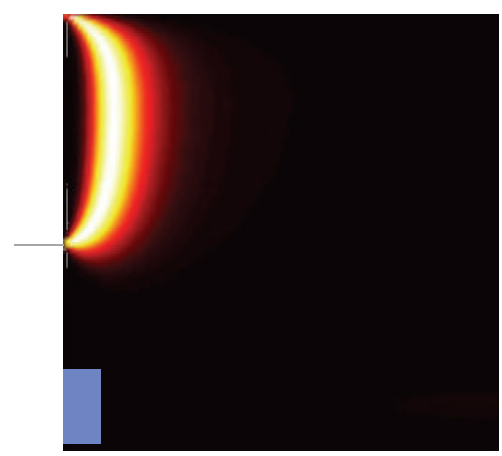

(i)

FIgURE 2: Two-dimensional $\beta^{2}(\rho, z)$ plot for different positions of the NP enforcing melted local ordering. One sees that NP affects position (they attract each other) of the melted finger-tip if they are close enough. Black colour indicates uniaxial ordering. Increasingly white colour reveals increasing degree of biaxiality. The distance $h-z_{f}$ describes the length of the finger-tip in absence of the NP.

droplets within a dispersion of water and phospholipid NPs of concentration $c$. We demonstrate that phospholipids assemble at NLC-water interface and that a critical concentration could trigger a structural transition within LC droplets. We first present experimental results. Then, we use a simple mesoscopic modelling to explain the observed transition.

5.1. Experimental Results. For the reference, we first studied nematic director configurations in micrometer-sized LC droplets ranging from $1 \mu \mathrm{m}$ to $10 \mu \mathrm{m}$ in absence of phospholipid molecules. All the droplets exhibited bipolar-type configurations. A representative bipolar NLC configuration is schematically sketched at the top of the last column in Figure 5. Such configurations were fingerprinted by timedependent interference textures revealing two surface point defects (boojums) at the poles of the droplets. Some representative interference textures are shown in Figures 5(a) and 5(b). Therefore, in absence of phospholipid molecules, an isotropic tangential anchoring condition is realized at the LC-water interface. Note that boojums exist only for strong enough tangential anchoring.

Next, we studied the impact of added phospholipids on nematic pattern within LC droplets. We studied samples with 


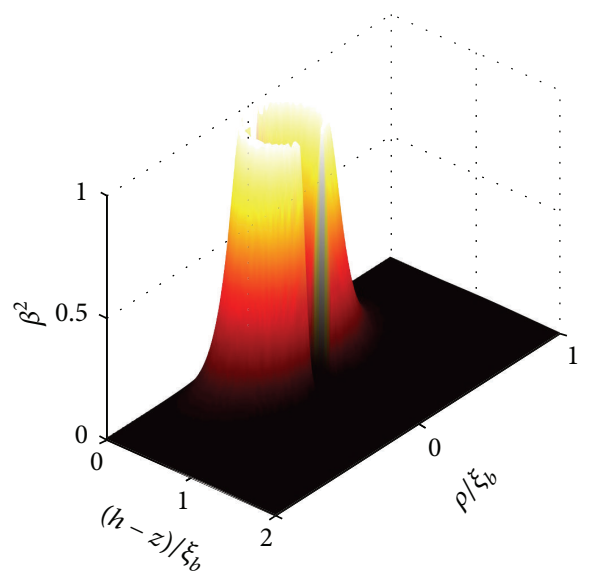

(a)

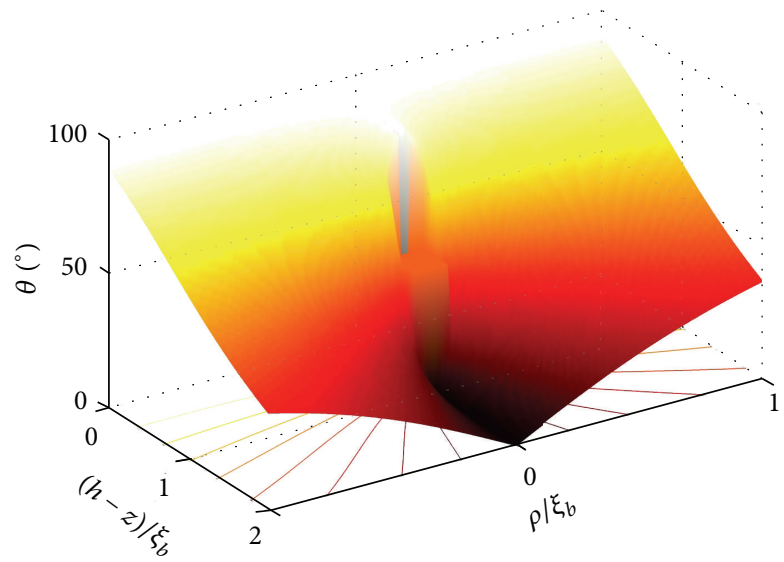

(b)

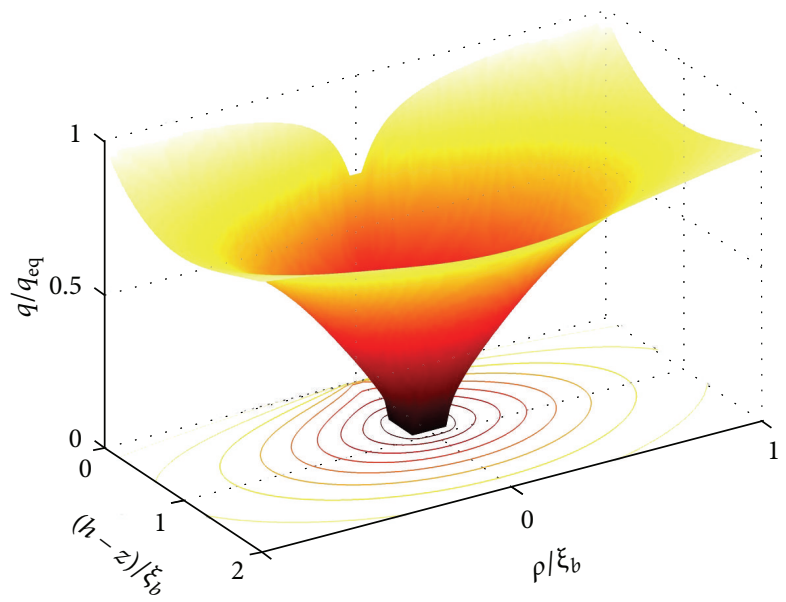

(c)

FIGURE 3: Two-dimensional plots showing degree of nematic ordering within the $\{\rho, z\}$ plane for a given position of a NP enforcing melted boundary condition. (a) $\beta^{2}(\rho, z)$, (b) $\theta(\rho, z)$, and (c) $q(\rho, z)=\sqrt{\operatorname{Tr} \mathbf{Q}^{2}}$, where $q_{\mathrm{eq}}$ describes the equilibrium value of $q . t=-8, r=\xi_{b} / 3$, and $h / \xi_{b}^{(0)}=10$.

phospholipid mass concentrations $c=10^{-7} \mathrm{~g} / \mathrm{mL}, 10^{-6} \mathrm{~g} / \mathrm{mL}$, $10^{-5} \mathrm{~g} / \mathrm{mL}, 10^{-4} \mathrm{~g} / \mathrm{mL}$, and $10^{-3} \mathrm{~g} / \mathrm{mL}$. The relationship with corresponding volume concentrations $\phi_{p}$ is given by $c=$ $\phi_{p} \rho_{p}$. Here, $\rho_{p} \sim \mathrm{g} / \mathrm{cm}^{3}$ stands for the phospholipid mass density. As previous studies revealed $[6,12,13]$, presence of phospholipids promotes homeotropic anchoring, enforcing radial-type structures. An example of spherically symmetric radial structure, which is stable in strong enough homeotropic anchoring, is sketched at the bottom of the last column of Figure 5. Viewed under crossed polarizers, radial structures typically exhibit four extinction bands with a cross-like appearance due to the presence of point defects (hedgehog) in the center of the structure. Representative interference textures are shown in Figures 5(c) and 5(d). For weaker anchoring strengths, the so-called preradial or escaped radial structure is commonly observed, exhibiting slightly different but comparable interference textures. Note that only rotating and translating droplets were analyzed to prevent observation of droplets interacting with a surface of glass plate. We observed images with a cross-like figure within droplets in crossed polarizers and hedgehog defect in the center of the droplet in the case of switched off analyzer (bright field) which was invariant with time. It corresponds to observation of radial configuration, whereas time-dependent figures indicated presence of bipolar one.

On increasing $c$, we observe increasing number of droplets possessing radial-type configurations. Henceforth, we use italic notations for all bipolar-type (bipolar) and radial-type (radial) nematic configurations. Relative number of radial and bipolar structures [14,15] in our samples is depicted in Figure 6. At $c=10^{-3} \mathrm{~g} / \mathrm{mL}$, almost all LC droplets ranging from $1 \mu \mathrm{m}$ to $8 \mu \mathrm{m}$ exhibit radial structures. On reducing $c$ below $10^{-4} \mathrm{~g} / \mathrm{mL}$, the number of radial structures markedly decrease, suggesting that the critical value of $c$ lies between $10^{-4} \mathrm{~g} / \mathrm{mL}$ and $10^{-5} \mathrm{~g} / \mathrm{mL}$. Such phospholipid driven structural behavior in water dispersed micrometersized droplets of azoxybenzene nematic liquid crystal $\mathrm{ZhK}$ 616 is quantitatively similar to the one observed using doubletailed phospholipids DOPC and DLPC in nematic 5CB droplets $[12,16]$. 


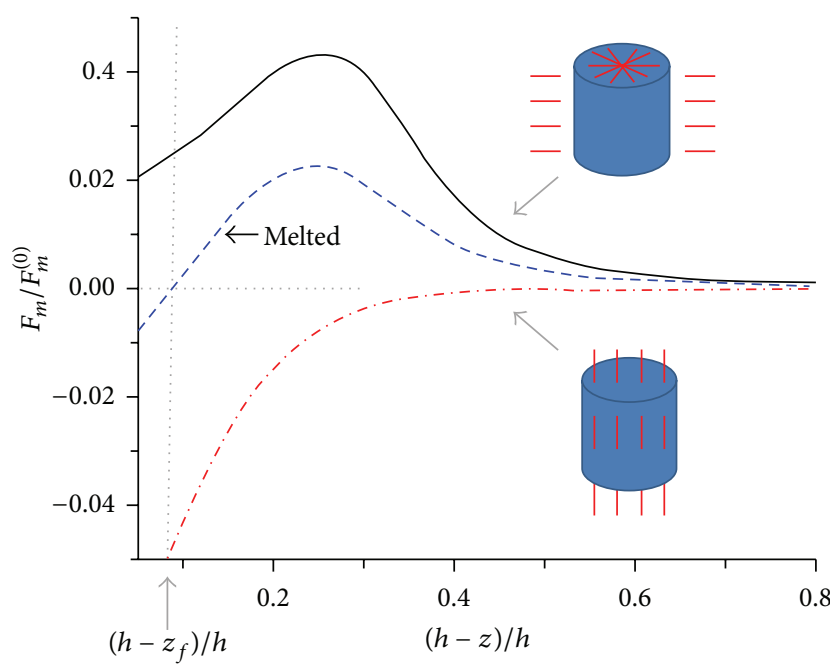

FIGURE 4: The mechanical force $F_{m}$ experienced by different surface treated NPs as a function of distance $z$ within the hybrid cell sketched in Figure 1(a). $F_{m}^{(0)}=\pi B^{4} h^{2} / 32 c^{3}$. The NPs locally enforce (i) radial, (ii) melted, or (iii) homogeneous local LC ordering. The corresponding forces for a given position of NP are marked with (i) full line, (ii) dashed line, and (iii) dash-dotted line. In the figure we also schematically sketch homogeneous and radial NP-induced local ordering tendency.

5.2. Theoretical Analysis. We next present a simple modelling to explain the observed structural transformation. For this purpose, we consider radial-bipolar structural transitions in a LC droplet of radius $R$ driven by interface assembled phospholipids.

Phospholipids possess flexible hydrophobic part and consequently they tend to be expelled from water. For large enough concentrations they could assemble into micelles what we did not observe. Therefore, we assume that most of the phospholipids are assembled at the surface of LC droplets where they locally enforce homeotropic anchoring.

In order to estimate critical concentration of phospholipids necessary to trigger bipolar-radial structural transition, we use our simple mesoscopic model [17] where for simplicity we consider only uniaxial ordering. We set that LC molecules at the interface enforce tangential anchoring, represented by the anchoring constant $w^{(\mathrm{NP})} S \equiv w_{t}>0$ in (5c). If a phospholipid is located at the interface, it locally enforces homeotropic anchoring, represented by $w^{(\mathrm{NP})} S \equiv-w_{h}<$ 0 . The number of LC and phospholipid molecules at the interface is given by $N_{t}$ and $N_{h}$, respectively. The total interfacial free energy of a LC droplet for a given structure is estimated by

$$
F_{i}^{\text {(structure) }} \sim-4 \pi R^{2} w_{\text {eff }} \overline{(\vec{n} \cdot \vec{v})^{2}}
$$

where we introduced the effective interface anchoring strength as

$$
w_{\text {eff }}=\frac{N_{h} a_{h} w_{h}-N_{t} a_{t} w_{t}}{4 \pi R^{2}}
$$

The quantities $a_{t}$ and $a_{h}$ describe the interface surface area occupied by LC and phospholipid molecule at the interface, respectively. The over-bar designates spatial average over the droplet surface interface. In addition, it holds

$$
N_{t} a_{t}+N_{h} a_{h} \sim 4 \pi R^{2}
$$

The volume free energy penalty of the "classical" radial and bipolar structure is estimated by [18]

$$
\begin{gathered}
F_{V}^{(\text {radial })} \sim 8 \pi K R+\frac{4 \pi \xi^{3}}{3}\left|f_{c}\right| \\
F_{V}^{\text {(bipolar })} \sim 5 \pi K R .
\end{gathered}
$$

Here, $K \sim L S_{\text {eq }}^{2}, K$ is the representative Frank elastic constant. The 2 nd term in (10) is due to the essentially melted core of the point defect within the radial structure.

The radial-bipolar structural transition is determined by the condition

$$
F^{(\text {radial })}=F^{(\text {bipolar })}
$$

where $F^{\text {(structure) }}=F_{V}^{\text {(structure) }}+F_{i}^{\text {(structure) }}$.

In radial and bipolar structures, we set $\overline{(\vec{n} \cdot \vec{v})^{2}} \sim 1$ and $\overline{(\vec{n} \cdot \vec{v})^{2}} \sim 0$, respectively. Furthermore, we assume that flexible phospholipids assemble within the topological defect. Consequently, the condensation free energy cost is reduced and we neglect the 2 nd term in (10). With this in mind we obtain an estimate for the critical number $N_{c}$ of phospholipids above which a radial configuration is favoured. One obtains

$$
N_{c} \sim \frac{4 \pi R^{2}}{a_{h}}\left(\frac{3}{4}+\frac{\sigma_{t}}{\sigma_{t}+\sigma_{h}}\right),
$$

where $\sigma_{h}=R w_{h} / K$ and $\sigma_{t}=R w_{t} / K$ are dimensionless numbers. For example, for $R=1 \mu \mathrm{m}, w_{t}=10^{-5} \mathrm{~J} / \mathrm{m}^{2}$, and $K=10^{-11} \mathrm{~J} / \mathrm{m}$, one obtains $\sigma_{t}=1$.

Next we introduce an average sample volume $V_{1}$ containing one spherical LC droplet. The volume concentration of LC molecules is thus

$$
\phi_{\mathrm{LC}}=\frac{4 \pi R^{3}}{\left(3 V_{1}\right)} .
$$
by

The volume concentration of phospholipids is then given

$$
\phi_{p}=\frac{N_{p} v_{p}}{V_{1}}=\frac{N_{p} \phi_{\mathrm{LC}} v_{p}}{\left(4 \pi R^{3} / 3\right)},
$$

where $N_{p}$ is the number of phospholipid molecules in $V_{1}$. In our approximate treatment, we set that all phospholipid molecules assemble at the interface. Therefore, $N_{t} \sim N_{p}$ and taking into account (13)-(15) a rough estimate for critical value $c_{c}$ of $c$ follows:

$$
c_{c} \sim \phi_{\mathrm{LC}} \rho_{p} \frac{3 v_{p}}{a_{h} R}\left(\frac{3}{4}+\frac{\sigma_{t}}{\sigma_{t}+\sigma_{h}}\right) .
$$


(a)

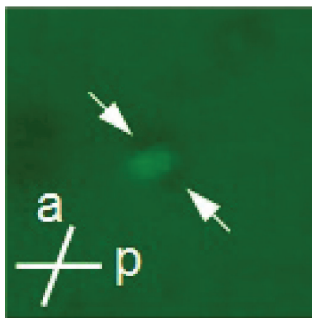

(b)

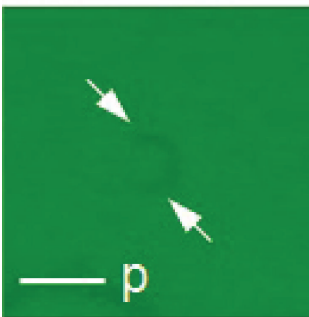

(c)

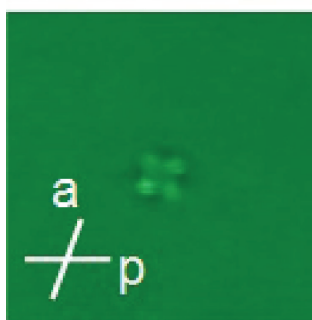

(d)

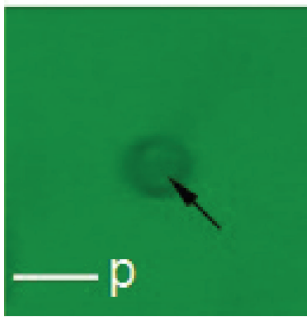

1
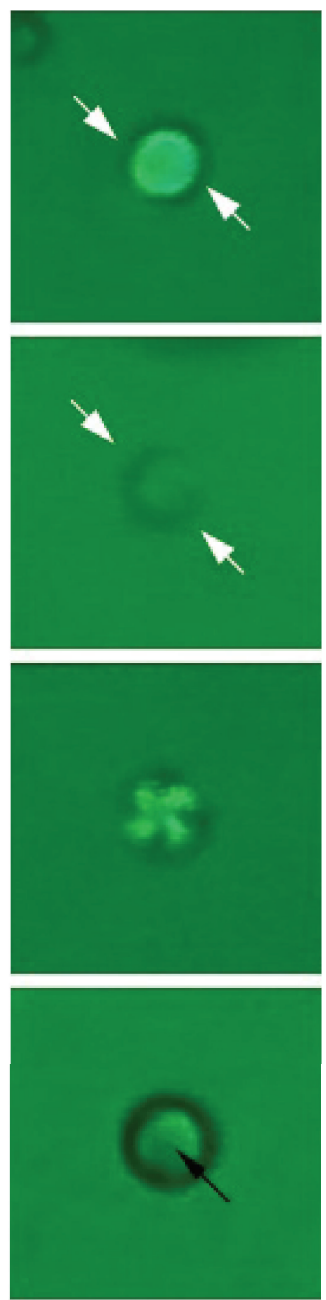

1.5
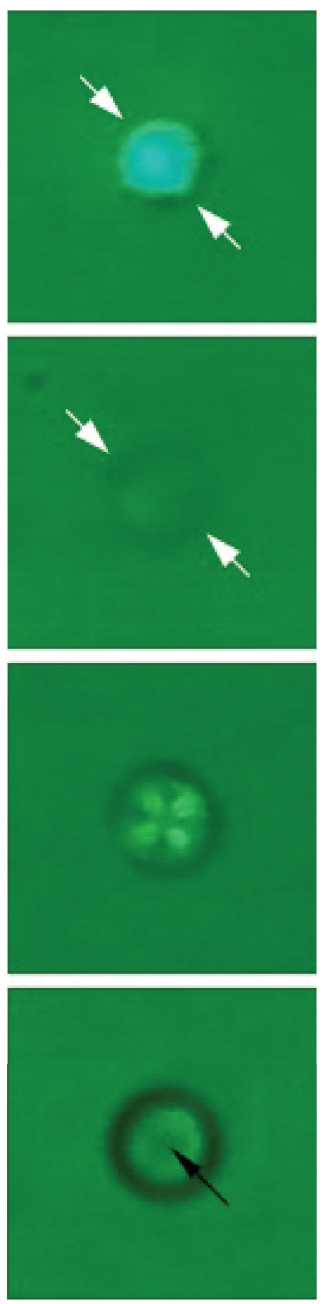

2
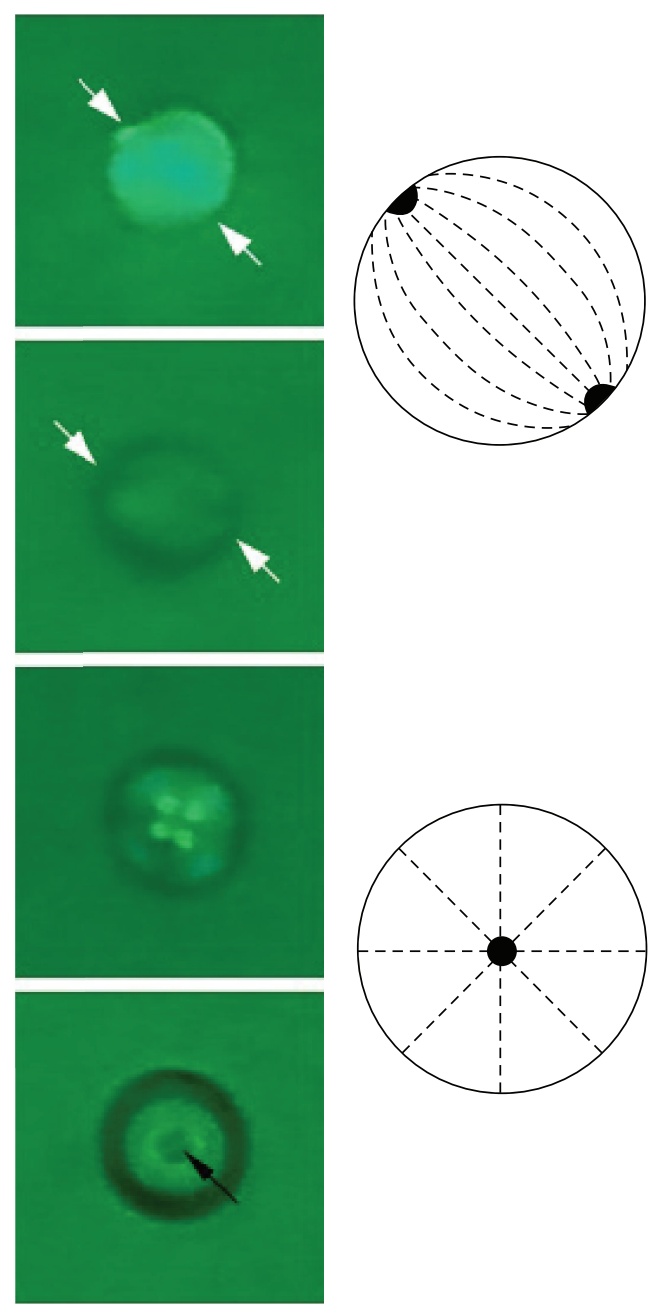

2.5
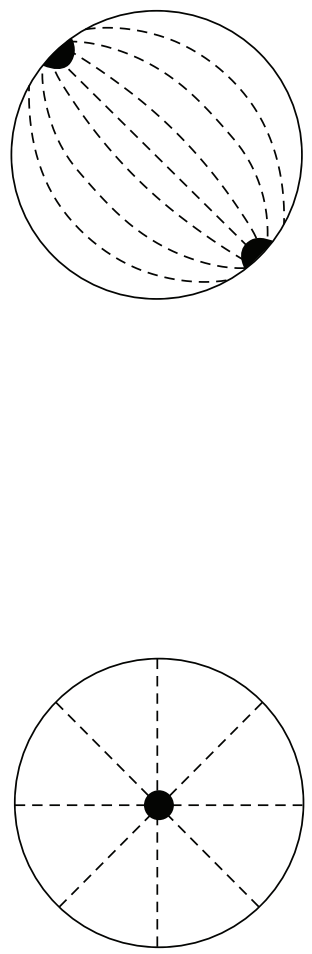

$R(\mu \mathrm{m})$

FIGURE 5: Interference textures of LC droplets of different radii obtained for crossed (a, c) and switched off analyser (b, d) in absence and presence of phospholipid: $(\mathrm{a}, \mathrm{b}) c=0,(\mathrm{c}, \mathrm{d}) c=10^{-3} \mathrm{~g} / \mathrm{mL}$. At the right panel we schematically plot typical bipolar (top) and radial (bottom) nematic structure. With white (black) arrows we mark positions of boojums (hedgehog) in bipolar (radial) structure. In (a) and (c) the polarizers are crossed at an angle of 70 degrees.

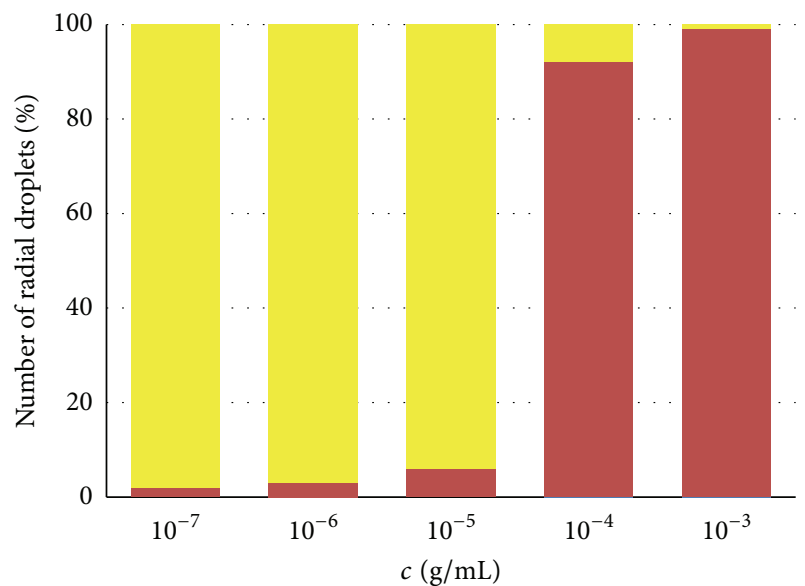

FIGURE 6: Relative number of LC droplets exhibiting radial (red) and bipolar (yellow) nematic structure as a function of $c$.
For a sensible set of parameters (i.e., $R \sim \mu \mathrm{m}, v_{h} / a_{h} \sim$ $30 \mathrm{~nm}, \sigma_{t} \sim \sigma_{h} \sim 10$, and $\phi_{\mathrm{LC}} \sim 10^{-3}$ ), we obtain $c_{c} \sim \phi_{p} \rho_{p} \sim$ $10^{-5} \mathrm{~g} / \mathrm{mL}$, where $\rho_{p} \sim \mathrm{g} / \mathrm{cm}^{3}$ stands for the mass density of phospholipids. In experiments, slightly larger concentrations are needed to trigger transitions, which signals that all phospholipids are not assembled at LC-water interface.

\section{Conclusions}

In the paper, we study interactions between NPs and nematic LC ordering. In the 1st part of the paper, we analyse numerically impact of NP's surface conditions (which could be controlled, e.g., by specific nanoparticle surface decoration method) on NP positioning within a spatially nonhomogeneous nematic LC ordering. Our aim was to identify a general robust mechanism which could potentially allow for controlled targeting of specific NPs to desired areas within 
a LC medium. The Landau-de Gennes approach in terms of the nematic tensor order parameter was used. For demonstrative purpose and to simplify numerical simulations, we studied a cylindrically shaped NP within a cylindrical cell enforcing hybrid orientational boundary conditions. We chose three qualitatively different NP's surface conditions in order to probe impact of different locally enforced topologies on NP targeting preferences. To be more specific, our chosen geometry possesses areas exhibiting essentially (i) radial, (ii) melted, and (iii) homogeneous nematic configuration. We inserted a NP imposing locally (i) radial, (ii) melted, or (iii) homogeneous nematic ordering. Our simulations reveal that NPs tend to migrate to regions which are most structurally compatible with their surface interaction imposed LC ordering tendency. Note that the chosen cylindrically shaped NP's geometry in our simulations plays a secondary role. For example, a spherically shaped NP would affect results only quantitatively. Our simulations identified a general robust targeting mechanism revealing that a NP tends to move into a region within which LC ordering matches best with NP's local LC ordering tendency as it is intuitively expected. In such a way, average elastic penalties are at least locally minimised.

In the second part of the paper, we demonstrate that controlled targeting of specific NPs to desired positions could efficiently trigger a global LC structural transition using a relatively small NP concentration. For this purpose we studied both experimentally and theoretically the impact of phospholipids on nematic ordering in nematic droplets dispersed in water containing concentration $c$ of phospholipids. For $c=0$, the droplets exhibited bipolar nematic configurations. On increasing $c$, we observed dramatic increase of droplets possessing radial structures above the threshold concentration $c_{c}>10^{-5} \mathrm{~g} / \mathrm{mL}$. We attributed this behaviour to adsorbed phospholipid molecules at the LC-water droplet interface, which locally enforce homeotropic anchoring. Therefore, for a high enough number of adsorbed phospholipid molecules, the effective anchoring condition at the interface can be changed. Consequently, nematic structural transition is triggered. Note that bipolar and radial interference textures are optically significantly different and the structural transition is triggered for a relatively low value of $c$. If combined, for example, with external electric or magnetic field, such system could be exploited for sensitive determination of relatively low concentrations of phospholipids in water. The mechanism demonstrated in our study could be exploited for development of sensitive sensors of specific NPs.

\section{Appendix}

\section{Parametrisation and Euler-Lagrange Equations}

For the chosen symmetry of the problem, it is convenient to parametrize the nematic tensor order parameter as

$$
\begin{aligned}
\mathbf{Q}= & -2 q_{1} \vec{e}_{\varphi} \otimes \vec{e}_{\varphi}+\left(q_{1}+q_{2}\right) \vec{e}_{\rho} \otimes \vec{e}_{\rho}+\left(q_{1}-q_{2}\right) \vec{e}_{z} \otimes \vec{e}_{z} \\
& +q_{3}\left(\vec{e}_{\rho} \otimes \vec{e}_{z}+\vec{e}_{z} \otimes \vec{e}_{\rho}\right) .
\end{aligned}
$$

Here, $q_{1}, q_{2}$, and $q_{3}$ are functions of $\rho$ and $z$. The order parameter $q_{3}$ measures departures of the eigenframe $\left\{\vec{e}_{1}, \vec{e}_{2}, \vec{e}_{3}\right\}$ of $\mathbf{Q}$ (see (1)) from the cylindrical coordinate system $\left\{\vec{e}_{\rho}, \vec{e}_{\varphi}, \vec{e}_{z}\right\}$. Namely, the two frames coincide for $q_{3}=0$. In general, the eigenvectors $\left\{\vec{e}_{1}, \vec{e}_{3}\right\}$ are rotated for an angle $\theta$ with respect to the pair $\left\{\vec{e}_{\rho}, \vec{e}_{z}\right\}$. It holds $\vec{e}_{1}=\vec{e}_{\rho} \sin \theta+\vec{e}_{z} \cos \theta, \vec{e}_{2}=\vec{e}_{\varphi}$, and $\vec{e}_{3}=-\vec{e}_{\rho} \cos \theta+\vec{e}_{z} \sin \theta$. Furthermore, the eigenvalues of $\mathbf{Q}$ are given by $s_{1}=-2 q_{1}, s_{2}=q_{1}-\kappa$, and $s_{3}=q_{1}+\kappa$, where $\kappa=\sqrt{q_{2}^{2}+q_{3}^{2}}$. The exchange between eigenvalues $s_{2}$ and $s_{3}$ occurs when $\kappa=0$.

We use the following dimensionless quantities in numerical treatment. We introduce the reduced temperature

$$
t=\frac{24 A_{0}\left(T-T_{*}\right) C}{B^{2}} .
$$

In terms of it the bulk equilibrium uniaxial order parameter reads

$$
S_{\mathrm{eq}}=s_{0}(1+\sqrt{1-t})
$$

where $s_{0}=B /(4 C)$. The biaxial correlation length is defined by

$$
\xi_{b}=\sqrt{\frac{4 \mathrm{LC}}{B^{2}(1+\sqrt{1-t})}}=\frac{\xi_{b}^{(0)}}{\sqrt{1+\sqrt{1-t}}}
$$

where $\xi_{b}^{(0)}$ is the bare, temperature independent biaxial correlation length. For typical LCs $\xi_{b}$ roughly ranges between $20 \mathrm{~nm}$ and $40 \mathrm{~nm}$ [11].

Furthermore, we implement rescaling $\mathbf{Q} \rightarrow \mathbf{Q} / s_{0}$ and scaling the spatial coordinates with respect to $h$. We henceforth retain the same name for scales order parameters $\rho, z, q_{1}, q_{2}$, and $q_{3}$ to avoid clutter. Using this scaling, we express dimensionless condensation and elastic free energy densities as

$$
\begin{aligned}
f_{c}= & \frac{t}{6}\left(3 q_{1}^{2}+q_{2}^{2}+q_{3}^{2}\right)-2 q_{1}\left(q_{2}^{2}+q_{3}^{2}-q_{1}^{2}\right) \\
& +\frac{1}{4}\left(3 q_{1}^{2}+q_{2}^{2}+q_{3}^{2}\right)^{2}, \\
f_{e}= & \left(\frac{\xi_{b}^{(0)}}{h}\right)^{2} \\
& \cdot\left(3\left|\nabla q_{1}\right|^{2}+\left|\nabla q_{2}\right|^{2}+\left|\nabla q_{3}\right|^{2}+\frac{\left(3 q_{1}+q_{2}\right)^{2}+q_{3}^{2}}{\rho^{2}}\right),
\end{aligned}
$$

and the gradient $\nabla$ is expressed by dimensionless space variables. 
Minimization of the free energy for given boundary conditions yields the bulk Euler-Lagrange equations for $q_{1}(\rho, z), q_{2}(\rho, z)$, and $q_{3}(\rho, z)$ :

$$
\begin{aligned}
& \left(\frac{\xi_{b}^{(0)}}{h}\right)^{2}\left(\Delta q_{1}-\frac{3 q_{1}+q_{2}}{\rho^{2}}\right)-\frac{t}{6} q_{1}+\frac{1}{3}\left(q_{2}^{2}+q_{3}^{2}-3 q_{1}^{2}\right) \\
& -\frac{q_{1}}{2}\left(3 q_{1}^{2}+q_{2}^{2}+q_{3}^{2}\right)=0 \\
& \left(\frac{\xi_{b}^{(0)}}{h}\right)^{2}\left(\Delta q_{2}-\frac{3 q_{1}+q_{2}}{\rho^{2}}\right)-\frac{t}{6} q_{2}+2 q_{1} q_{2} \\
& -\frac{q_{2}}{2}\left(3 q_{1}^{2}+q_{2}^{2}+q_{3}^{2}\right)=0 \\
& \left(\frac{\xi_{b}^{(0)}}{h}\right)^{2}\left(\Delta q_{3}-\frac{q_{3}}{\rho^{2}}\right)-\frac{t}{6} q_{3}+2 q_{1} q_{3} \\
& -\frac{q_{3}}{2}\left(3 q_{1}^{2}+q_{2}^{2}+q_{3}^{2}\right)=0
\end{aligned}
$$

Here, $\Delta=\partial^{2} / \partial \rho^{2}+(1 / \rho)(\partial / \partial \rho)+\partial^{2} / \partial z^{2}$ is the Laplace operator. Note that a positive uniaxial order corresponding to the nematic director field $\vec{n}=\vec{e}_{\rho} \sin \theta+\vec{e}_{z} \cos \theta$ and $S=S_{\text {eq }}$ corresponds to

$$
\begin{gathered}
q_{1}=\frac{1+\sqrt{1-t}}{6}, \quad q_{2}=\frac{1+\sqrt{1-t}}{2} \cos (2 \theta), \\
q_{3}=\frac{1+\sqrt{1-t}}{2} \sin (2 \theta) .
\end{gathered}
$$

The equilibrium differential equations are solved by standard relaxation method.

\section{Conflict of Interests}

The authors of the paper declare that there is no conflict of interests regarding the publication of this paper.

\section{Acknowledgments}

This work was partially supported by the Ministry of Education and Science of the Russian Federation (Grant nos. 3.1921.2014/K and 14.B37.21.1511), the RFBR (Project no. 1403-31773 mol_a), and MGUPI (PL-044).

\section{References}

[1] H. Kikuchi, M. Yokota, Y. Hisakado, H. Yang, and T. Kajiyama, "Polymer-stabilized liquid crystal blue phases," Nature Materials, vol. 1, no. 1, pp. 64-68, 2002.

[2] E. Karatairi, B. Rožič, Z. Kutnjak et al., "Nanoparticle-induced widening of the temperature range of liquid-crystalline blue phases," Physical Review E-Statistical, Nonlinear, and Soft Matter Physics, vol. 81, no. 4, Article ID 041703, 2010.

[3] V. J. Anderson, E. M. Terentjev, S. P. Meeker, J. Crain, and W. C. K. Poon, "Cellular solid behaviour of liquid crystal colloids 1.
Phase separation and morphology," European Physical Journal E, vol. 4, no. 1, pp. 11-20, 2001.

[4] P. G. de Gennes and J. Prost, The Physics of Liquid Crystals, Oxford University Press, Oxford, UK, 1993.

[5] P. Palffy-muhoray, E. C. Gartland, and J. R. Kelly, "A new configurational transition in inhomogeneous nematics," Liquid Crystals, vol. 16, no. 4, pp. 713-718, 1994.

[6] G. E. Volovik and O. D. Lavrentovich, "The topological dynamics of defects-boojums in nematic drops," Zhurnal Eksperimental'noi i Teoreticheskoi Fiziki, vol. 85, no. 6, pp. 1997-2010, 1983.

[7] M. Kleman and O. D. Lavrentovich, Soft Matter Physics: An Introduction, Springer, 2003.

[8] S. Kralj, R. Rosso, and E. G. Virga, "Finite-size effects on order reconstruction around nematic defects," Physical Review EStatistical, Nonlinear, and Soft Matter Physics, vol. 81, no. 2, Article ID 021702, 2010.

[9] P. Kaiser, W. Wiese, and S. Hess, "Stability and instability of an uniaxial alignment against biaxial distortions in the isotropic and nematic phases of liquid crystals," Journal of NonEquilibrium Thermodynamics, vol. 17, no. 2, pp. 153-169, 1992.

[10] S. Kralj, R. Rosso, and E. G. Virga, "Fingered core structure of nematic boojums," Physical Review E: Statistical, Nonlinear, and Soft Matter Physics, vol. 78, no. 3, Article ID 031701, 2008.

[11] G. Lombardo, A. Amoddeo, R. Hamdi, H. Ayeb, and R. Barberi, "Biaxial surface order dynamics in calamitic nematics," European Physical Journal E, vol. 35, no. 5, article 32, 2012.

[12] I.-H. Lin, D. S. Miller, P. J. Bertics, C. J. Murphy, J. J. de Pablo, and N. L. Abbott, "Endotoxin-induced structural transformations in liquid crystalline droplets," Science, vol. 332, no. 6035, pp. 1297$1300,2011$.

[13] O. D. Lavrentovich, "Topological defects in dispersed liquid crystals, or words and worlds around liquid crystal drops," Liquid Crystals, vol. 24, no. 1, pp. 117-125, 1998.

[14] P. S. Drzaic, "Droplet density, droplet size, and wavelength effects in PDLC light scattering," Molecular Crystals and Liquid Crystals Science and Technology. Section A. Molecular Crystals and Liquid Crystals, vol. 261, no. 1, pp. 383-392, 1995.

[15] O. O. Prischepa, A. V. Shabanov, and V. Y. Zyryanov, "Director configurations within nematic droplets doped by lecithin," Molecular Crystals and Liquid Crystals, vol. 438, no. 1, pp. 141150, 2005.

[16] D. S. Miller and N. L. Abbott, "Influence of droplet size, pH and ionic strength on endotoxin-triggered ordering transitions in liquid crystalline droplets," Soft Matter, vol. 9, no. 2, pp. 374382, 2013.

[17] A. V. Dubtsov, S. V. Pasechnik, D. V. Shmeliova, and S. Kralj, "Light and phospholipid driven structural transitions in nematic microdroplets," Applied Physics Letters, vol. 105, no. 15, Article ID 151606, 2014.

[18] E. Dubois-Violette and E. Parodi, "Émulsions nématiques. effets de champ magnétiques et effets piézoélectriques," Le Journal de Physique Colloques, vol. 30, no. C4, pp. C4-57-C4-64, 1969. 

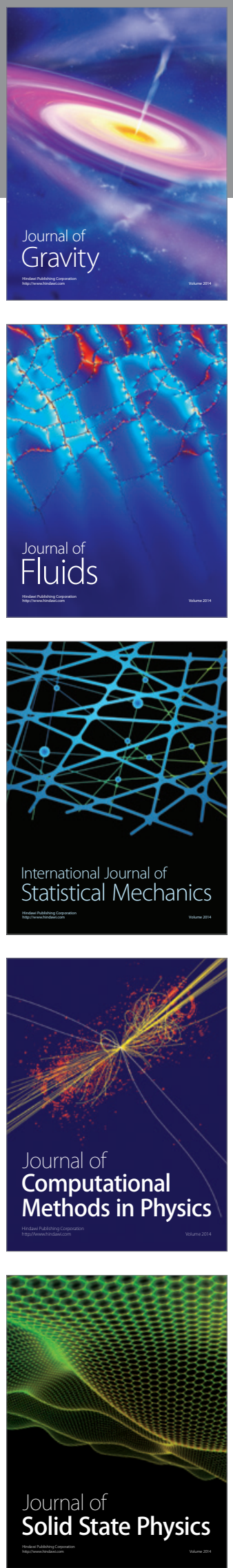

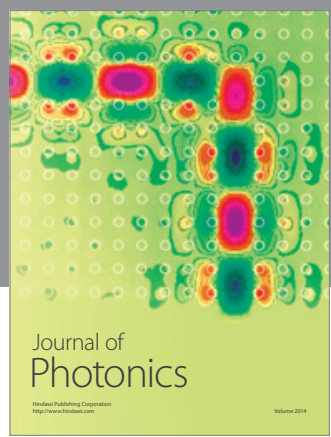

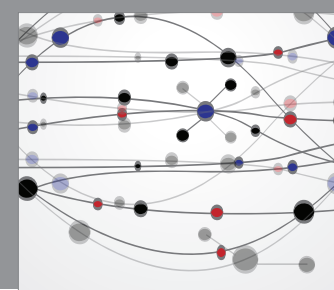

The Scientific World Journal

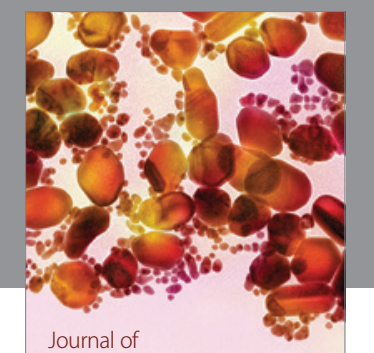

Soft Matter
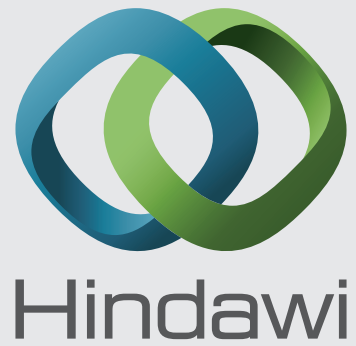

Submit your manuscripts at

http://www.hindawi.com
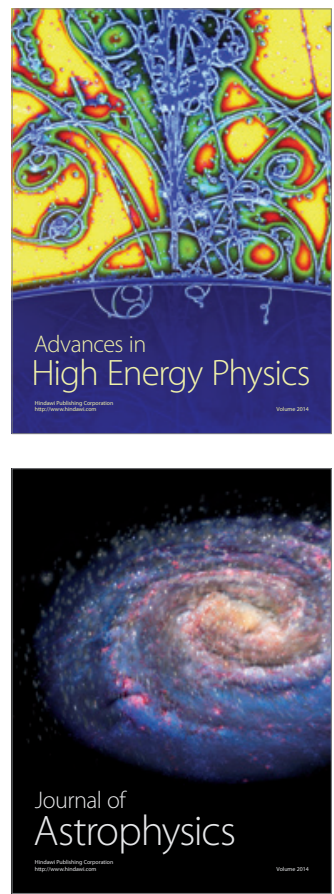
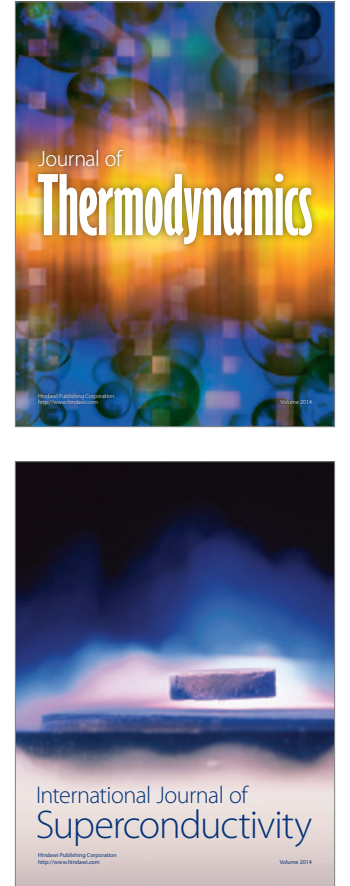
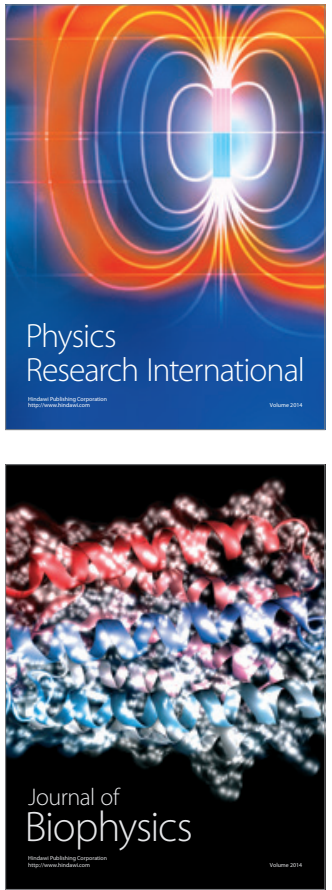
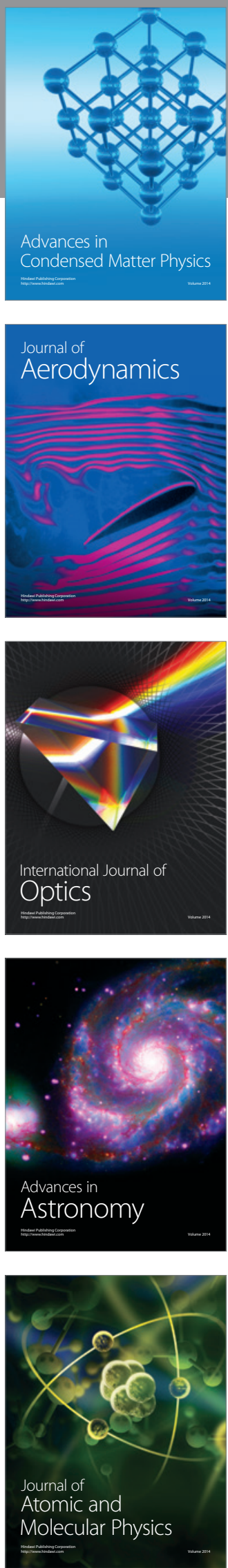